\title{
NOISE-ASSISTED MULTIVARIATE VARIATIONAL MODE DECOMPOSITION
}

\author{
Charilaos A. Zisou $\quad$ Georgios K. Apostolidis ${ }^{\star} \quad$ Leontios J. Hadjileontiadis $^{\star \dagger}$ \\ * School of Electrical \& Computer Eng., Aristotle University of Thessaloniki, GR-54124, Greece \\ ${ }^{\dagger}$ Department of Electrical \& Computer Eng., Khalifa University, PO Box 127788, UAE
}

\begin{abstract}
The variational mode decomposition (VMD) is a widely applied optimization-based method, which analyzes nonstationary signals concurrently. Correspondingly, its recently proposed multivariate extension, i.e., MVMD, has shown great potentials in analyzing multichannel signals. However, the requirement of presetting the number of extracted components $\mathrm{K}$ diminishes the analytic property of both VMD and MVMD methods. This work combines MVMD with the noise injection paradigm to propose an efficient alternative for both VMD and MVMD, i.e., the noise-assisted MVMD (NA-MVMD), that aims at relaxing the requirement of presetting $\mathrm{K}$, as well as improving the quality of the resulting decomposition. The noise is injected by adding noise variables/channels to the initial signal to excite the filter bank property of VMD/MVMD on white Gaussian noise. Moreover, an alternative approach of updating center frequencies is proposed, which uses the centroid of the generalized cross-spectrum instead of a simple average of the individual spectral centroids, showing faster convergence. The NA-MVMD is applied to both univariate and multivariate synthetic signals, showing improved analytical ability, noise intolerance, and less sensitivity in selecting the $\mathrm{K}$ parameter.
\end{abstract}

Index Terms - multivariate analysis, variational mode decomposition, multichannel signals, non-stationary signals

\section{INTRODUCTION}

Over the last two decades, data-driven signal decomposition methods have proven to be useful tools in non-stationary signal analysis and have been widely used in a plethora of problems. The objective of these methods is to output a set of components whose Hilbert transform accurately represents the instantaneous dynamics, i.e. instantaneous amplitude and frequency, of an input signal. It is well known that the Hilbert transform can disentangle the instantaneous amplitude and frequency, only in the case of non-overlapping frequency spectra (Bedrosian theorem) [1]. Thus all existing data-driven methods aim at extracting components with slow-varying

Thanks to Projects: Care4MyHeart with ADEK Award Number AARE18-135 and PROTEIN Grant no.817732 within the H2020 Research and Innovation Program for funding this work. (C. Zisou and G. Apostolidis contributed equally to this work.). Code: https://github.com/chariszisou amplitudes, i.e. narrow-band signals. The very first method proposed was the empirical mode decomposition (EMD) [2] which analyzes an input signal into a set of narrow-band components via an iterative sifting process which is driven by the signal's extrema and does not require any a priori defined basis. More recently, a different data-driven approach that has drawn significant attention is the variational mode decomposition (VMD) which formulates an optimization problem and minimizes the collective bandwidth (CBW) of the signal's components subject to the full reconstruction constraint. Several data-driven signal decomposition methods can be found in the bibliography, many of which have been proposed as extensions of the original EMD algorithm [3, 4, 5, 6] as well as alternative approaches, such as the iterative filtering [7], the empirical wavelet transform [8], the synchrosqueezed transform [9] and the swarm decomposition [10]. Typically, the aforementioned methods are designed to analyze univariate signals. Nevertheless, multivariate non-stationary signal analysis using data-driven methods attracts great interest from the scientific areas related to multichannel/multisensor signal processing. Although a multivariate signal can be processed channel-wise, the alignment of frequencies across channels, i.e. the mode-alignment, is not guaranteed. Thus, multivariate extensions of the univariate data-driven decomposition methods have been proposed, e.g. the multivariate EMD (MEMD) [11, 12] and the multivariate VMD (MVDM) [13], among others $[14,15,16]$.

This work focuses on improving the VMD and MVMD methods. The main concept of MVMD, similarly to VMD, is to formulate an optimization problem to extract a collection of components that exhibit minimum CBW subject to full reconstruction of the signal of every channel of the input multivariate data. At every optimization step an ensemble of components for each channel $k$ is resulted via applying bandpass filtering around floating dominant frequencies $\omega_{k}$, which are updated online during the process. The floating dominant frequency for each component is calculated as the average of the spectral centroids across all channels.

The main issue of both VMD and MVMD methods is that they are highly parameter-sensitive. Specifically, the decomposition result is heavily influenced by the number of components $K$ that must be defined beforehand and arbitrary presetting of $K$ may cause mode splitting. Thus, scholars have 
approached this problem with a focus on estimating the optimal parameter $K[17,18,19,20]$. The aim of this work is not to propose another parameter $K$ estimation approach, but to make the original methods independent from $K$, under certain circumstances. An additional objective is to improve the original methods' decomposition capability, i.e. to enhance the resulting component orthogonality. To meet these objectives, we exploit the filter bank property of both VMD and MVMD on white Gaussian noise (WGN) [13, 21].

The new method, the noise-assisted MVMD (NA-MVMD) combines the MVMD with the noise injection paradigm proposed in [6]. In short, NA-MVMD creates a noise injected multivariate signal by adding new channels that consist of independent realizations of WGN to the input data channels and then applies the standard MVMD to the new multivariate signal. Finally, in the update of the floating dominant frequencies, the average spectral centroid across all channels is replaced by the spectral centroid of the generalized cross-spectrum [22]. To prove the potential of NA-MVMD, experiments are conducted using both univariate and multivariate synthetic signals.

\section{MULTIVARIATE VMD}

The crux of MVMD [13] is to analyze a multivariate input signal $\mathbf{x}(t)=\left[x_{1}(t), x_{2}(t), \cdots, x_{C}(t)\right]$ containing $C$ number of data channels into a predefined number $K$ of multivariate components $\mathbf{u}_{k}(t)$, i.e.

$$
\mathbf{x}(t)=\sum_{k=1}^{K} \mathbf{u}_{k}(t),
$$

where $\mathbf{u}_{k}(t)=\left[u_{k, 1}(t), u_{k, 2}(t), \cdots, u_{k, C}(t)\right]$. The goal of MVMD is to extract an ensemble of multivariate components $\left\{\mathbf{u}_{k}(t)\right\}_{k=1}^{K}$ the CBW of which, is minimum across all channels. Thus, the optimization cost function [13] is

$$
g\left(\left\{u_{k, c}\right\},\left\{\omega_{k}\right\}\right)=\sum_{k=1}^{K} \sum_{C=1}^{C}\left\|\partial_{t}\left[u_{k, c}^{a}(t) \cdot \exp ^{-j \omega_{k} t}\right]\right\|_{2}^{2},
$$

where $u_{k, c}^{a}(t)$ is the analytic modulated signal that represents the $k^{t h}$ oscillatory mode of channel $c$. The MVMD output is the solution of the constraint optimization problem,

$$
\begin{gathered}
\min _{\left\{u_{k, c}\right\},\left\{\omega_{k}\right\}} g\left(\left\{u_{k, c}\right\},\left\{\omega_{k}\right\}\right), \\
\text { subject to } x_{c}(t)=\sum_{k=1}^{K} u_{k, c}(t) .
\end{gathered}
$$

Next, the optimization problem in (3) is converted to an unconstrained problem using the method of Lagrange multipliers. The corresponding Lagrange function is

$$
\begin{gathered}
\mathcal{L}\left(\left\{u_{k, c}\right\},\left\{\omega_{k}\right\}, \lambda_{c}\right)=\alpha \cdot g\left(\left\{u_{k, c}\right\},\left\{\omega_{k}\right\}\right)+ \\
+\sum_{c=1}^{C}\left\|e_{c}(t)\right\|_{2}^{2}+\sum_{c=1}^{C}\left\langle\lambda_{c}(t), e_{c}(t)\right\rangle, \\
e_{c}(t)=x_{c}(t)-\sum_{k=1}^{K} u_{k, c}(t)
\end{gathered}
$$

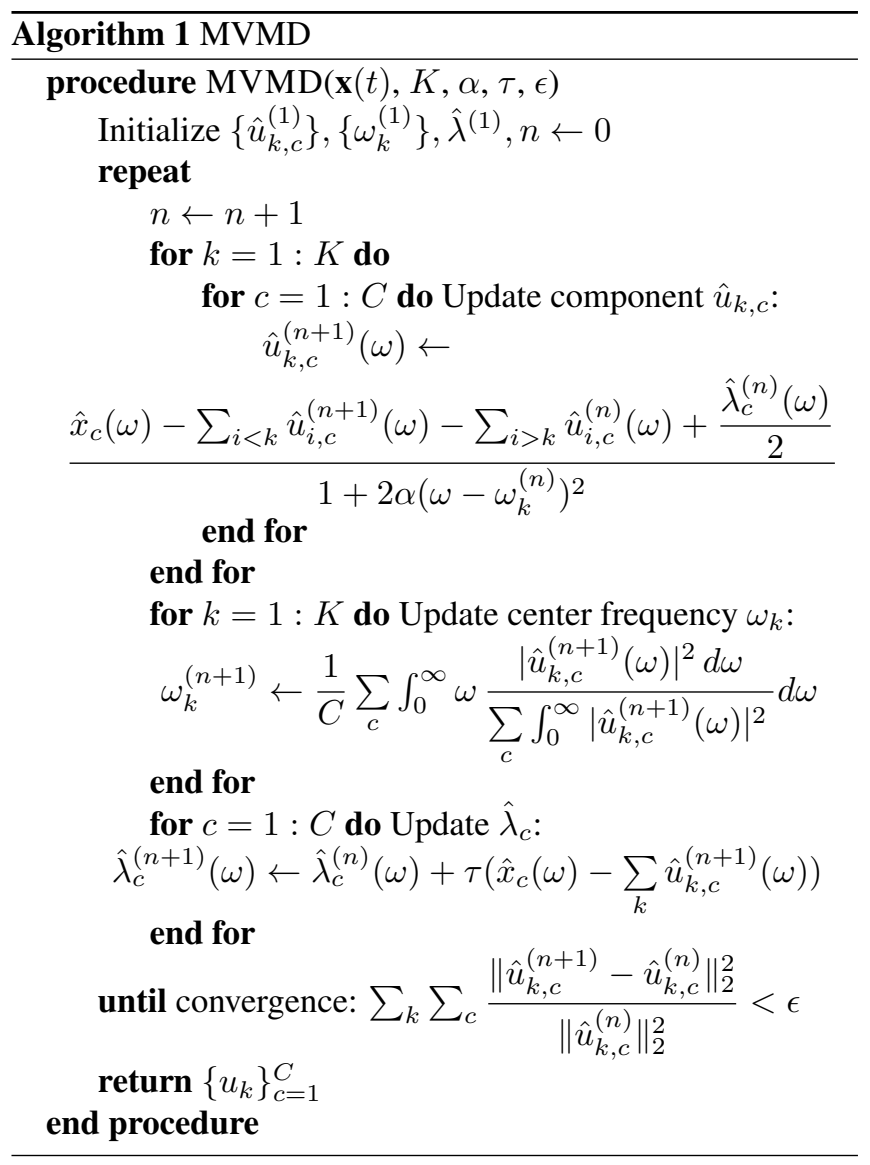

where $\alpha$ is a balancing parameter, $\left\{\lambda_{c}(t)\right\}_{c=1}^{C}$ are the Lagrange multipliers and $e_{c}(t)$ is the reconstruction error of the $c^{t h}$ channel. In MVMD, (4) is solved using the alternate direction method of multipliers (ADMM) approach [23], and the complete optimization problem is solved as a sequence of iterative sub-optimization problems. Details regarding the formulation of ADMM for solving MVMD optimization problem can be found in [13].

In practice, the algorithm that implements the MVMD method is divided in three parts, i.e the component update, the central frequencies update and the Langrange multipliers update, which are executed iteratively until they reach a convergence criterion. The MVMD algorithm is presented in Algorithm 1, where $K, \alpha, \tau$ and $\epsilon$ are the predefined number of components, the balancing parameter, the Lagrange update parameter and the convergence tolerance. Moreover, the hat symbol $\left({ }^{\circ}\right)$ indicates the Fourier transform and the parenthesis superscript denotes the iteration number. In detail, the component update is based on a second-order band-pass filtering of the current residual regularized by $\hat{\lambda}_{c}$ with center frequency $\omega_{k}$, which is common across all channels. Furthermore, the center frequencies update is based on the averaged spectral centroids. Finally, the algorithm terminates when consecutive sets of components remain unchanged. It is worth noting that for $C=1$ the MVMD is reduced to VMD [21]. 


\section{NOISE-ASSISTED MVMD}

The NA-MVMD method is a refinement of MVMD. The new elements that NA-MVMD includes are the adoption of the noise injection paradigm proposed in [6] and the substitution of the center frequency update calculation. The NA-MVMD approach can be applied to both univariate and multivariate signals. The noise injection aims at exciting the filter bank character of the original MVMD method to provide a distinct processing behavior regardless of the input signal characteristics. The noise injection is performed by adding channels that consist of independent realizations of WGN,

$$
\mathbf{x}^{\prime}(t)=\left[\mathbf{x}(t), \eta_{1}(t), \cdots, \eta_{\mathcal{N}}(t)\right]
$$

where $\mathbf{x}^{\prime}(t)$ is the new multivariate signal, $\mathbf{x}(t)$ is the input univariate or multivariate signal and $\eta_{i}(t), \forall i=1, \cdots, \mathcal{N}$, are WGN signals. Then, $\mathbf{x}^{\prime}(t)$ replaces $\mathbf{x}(t)$ in Algorithm 1. Moreover, the center frequencies update part of MVMD changes from the average of spectral centroids across all channels to the centroid of the generalized cross-spectrum (GCS) [22]. For a set of $N$ signals $\left\{z_{i}(t)\right\}_{i=1}^{N}$, the GCS is

$$
\begin{gathered}
\gamma(\omega)=(1 /(N-1))\left(\Lambda_{\max }\left(\boldsymbol{\Sigma}_{z}(\omega)\right)-1\right), \\
\boldsymbol{\Sigma}_{z}(\omega)=\left[\begin{array}{ccc}
S_{z_{1} z_{1}}(\omega) & \ldots & S_{z_{1} z_{N}}(\omega) \\
\vdots & \ddots & \vdots \\
S_{z_{N} z_{1}}(\omega) & \ldots & S_{z_{N} z_{N}}(\omega)
\end{array}\right]
\end{gathered}
$$

where $\boldsymbol{\Sigma}_{z}(\omega)$ contains all the pairwise complex crossspectra, $S_{z i, z j}(\omega) \forall i, j=1, \cdots, N$, and $\Lambda_{\max }\left(\boldsymbol{\Sigma}_{x}(\omega)\right)$ is the largest eigenvalue of the matrix $\boldsymbol{\Sigma}_{z}(\omega)$. It is evident that for the case of $N=2$ the GCS is reduced to the standard cross-spectrum. Now, let $\gamma_{k}(\omega)$ be the GCS of the set of $k^{t h}$-level components $\left\{\hat{u}_{k, c}(\omega)\right\}_{c=1}^{C}$ at an arbitrary iteration of Algorithm 1 the update of center frequency $\omega_{k}$ becomes

$$
\omega_{k}=\int_{0}^{\infty} \omega \frac{\gamma_{k}(\omega)}{\int_{0}^{\infty} \gamma_{k}(\omega) d \omega} d \omega .
$$

The reason for this replacement is that the new approach is a more elegant representation of the components' collective spectrum and it is a direct extension of the VMD method.

\section{EXPERIMENTAL RESULTS}

To evaluate its performance, NA-MVMD was applied, along with VMD and MVMD, to non-stationary univariate and multivariate synthetic signals.

\subsection{Dependence of the parameter $K$, influence of noise and orthogonality property}

The performance of the NA-MVMD method was tested on synthetic univariate multi-component signals and compared with the well-known VMD method. Particular emphasis is given on testing the influence of the parameter $K$, i.e. the number of extracted components as well as investigating the orthogonality condition.

The synthetic signals generated for this experiment were realizations of a stochastic class of non-stationary multicomponent signals. This class of signals was defined as

$$
\begin{gathered}
s(n)=\sum_{m=1}^{M} s_{m}(n)+\eta(n), n \in \mathbb{Z}, \\
s_{m}(n)=w_{L_{m}}\left(n-d_{m}\right) \cdot A_{m} \cdot \cos \left(\omega_{m} n\right), n \in \mathbb{Z},
\end{gathered}
$$

where $M$ is the number of generated components, $\eta(n)$ is the added WGN, $w_{L_{m}}\left[n-d_{m}\right]$ is a Hanning window of time location $d_{m}$ and length $L_{m}, A_{m}$ is the amplitude and $\omega_{m}$ is the frequency of $m^{\text {th }}$ component. For this experiment $M, d_{m}$ and $L_{m}$ were fixed, i.e. $M=3, d_{m}=\{250,125,375\}, L_{m}=$ $\{500,125,100\}$, whereas, $A_{m}$ and $\omega_{m}$ were randomly drawn from uniform distributions, i.e. $P D F\left(A_{m}\right)=U(0.5,1.5)$ and $P D F\left(\omega_{m}\right)=U(0, \pi), \forall m$.

In order to compare both methods in a generalized manner, multiple simulations were executed for a sufficient amount of iterations, i.e. 500, for three different signal-tonoise ratios (SNR). Every iteration consisted of the following steps: 1) a multi-component signal (10) was generated, 2) the signal was decomposed for different values of $K$, i.e. $K=\{3: 10\}$ using both VMD and NA-MVMD, 3) the decompositions were characterized as successful or not, and, finally, 4) their orthogonality index (OI) was calculated. Success was defined as a binary variable (1/0). A decomposition was classified as successful, if all input components (11) appeared exactly once in the set of the output components. The absence or the splitting of a component was considered incorrect. This definition is very strict, e.g. if 2 out of 3 components are correctly extracted, the success is set to 0 . At each scenario, after all iterations, the success rate $S R$ was calculated as the number of successful decompositions divided by the total number of repetitions. Furthermore, the OI was calculated based on the definition in [2], where for an input signal $s(n)$ and output components $u_{m}(n), \forall m=1, \cdots, M$,

$$
O I=\sum_{n}\left(\sum_{i=1}^{M} \sum_{j=1}^{M} u_{i}(n) \cdot u_{j}(n) / s^{2}(n)\right)
$$

Except for $K$, the rest of Algorithm 1 parameters are set as $\alpha=1000, \tau=10^{-4}$ and $\epsilon=10^{-8}$. Following (6), one assisting noise channel is added. Fig. 1 illustrates the analysis of an excerpt signal from the simulations, revealing the main shortcoming of VMD, i.e. the mode splitting.

Fig. 2 depicts the success of both the proposed and the original method on decomposing the generated synthetic signals versus the parameter $K$. For high values of SNR, VMD shows a spike around $K=3$, highlighting the necessity of choosing the correct number of $K$ a priori, since VMD fails at other $K$. In contrary, the NA-MVMD method successfully analyzes signals independently of $K$ making the a priory selection of $K$ less restrictive. Moreover, NA-MVMD behavior remains unchanged for different values of SNR showing good noise intolerance. 


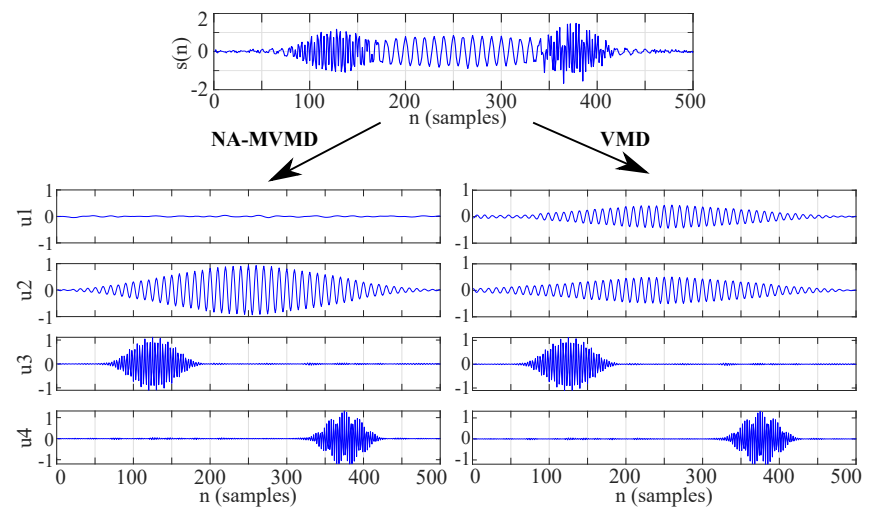

Fig. 1. An excerpt signal from the experiment with $S N R=$ $20 \mathrm{~dB}, \omega_{m}=\{0.2 \pi, 0.6 \pi, 0.7 \pi\}$ and $A_{m}=\{0.9,1.2,1.4\}$ and its extracted components applying the NA-MVMD and the VMD methods. This example shows the mode splitting that occurs in VMD (see components $u_{1}$ and $u_{2}$ ) and, the respective rectification using NA-MVMD.

Table 1 depicts the OI versus SNR for both NA-MVMD and VMD methods for those $K$ that each method has the best SR. It is evident that NA-MVMD performs better in terms of the orthogonality property.

Table 1. The $\mathrm{OI} \times 10^{-2}$ (mean and standard deviation) of NAMVMD and VMD for different SNR, using the best performing $\mathrm{K}$, i.e $\mathrm{K}=8$ for the NA-MVMD and $\mathrm{K}=3$ for the VMD.

\begin{tabular}{c|c|c|c}
\hline SNR (dB) & $\mathbf{2 0}$ & $\mathbf{1 5}$ & $\mathbf{1 0}$ \\
\hline \hline NA-MVMD & $0.06 \pm 0.03$ & $0.07 \pm 0.03$ & $0.1 \pm 0.03$ \\
\hline VMD & $0.17 \pm 0.13$ & $0.13 \pm 0.08$ & $0.17 \pm 0.08$ \\
\hline
\end{tabular}

\subsection{Convergence Analysis}

In this experiment, a comparative evaluation regarding the center frequency update (see Algorithm 1) was conducted. The comparison involved two approaches, the averagingbased and the GCS-based, i.e. the original and the proposed one, respectively. For this purpose a synthetic trivariate signal was generated, $\mathbf{x}(n)=\left[x_{1}(n), x_{2}(n), x_{3}(n)\right]+\eta(n)$ with,

$$
\begin{aligned}
& x_{1}(n)=0.5 \sin \left(\omega_{1} n\right)+0.2 \sin \left(\omega_{2} n\right)+0.1 \sin \left(\omega_{3} n\right), \\
& x_{2}(n)=0.45 \cos \left(\omega_{1} n\right)+0.3 \sin \left(\omega_{3} n\right)+0.2 \sin \left(\omega_{4} n\right), \\
& x_{3}(n)=0.4 \sin \left(\omega_{1} n\right)+0.25 \sin \left(\omega_{2} n\right),
\end{aligned}
$$

where $\omega_{1}=0.1 \pi, \omega_{2}=0.2 \pi, \omega_{3}=0.3 \pi, \omega_{4}=0.8 \pi$, $\eta(n)$ is WGN and $n \in \mathbb{Z}$. MVMD was applied using both approaches, for different values of SNR. At each SNR level, the process was repeated for 100 iterations and the number of steps until convergence was monitored. The results are presented in Table 2 showing that the proposed approach provides faster convergence especially in high noise levels. This is explained by the fact that the averaging, as a smoothing operation, is in general less sensitive in cases of high variance.

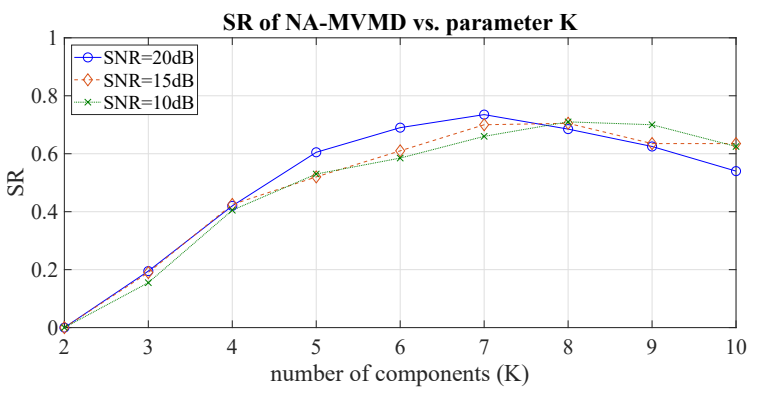

(a)

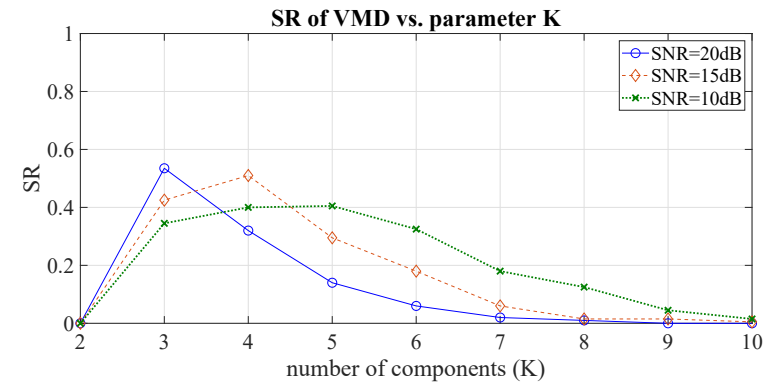

(b)

Fig. 2. The SR of NA-MVMD and VMD with respect to K.

Table 2. Median and standard deviation of the total number of steps until convergence for the GCS-based (proposed) and the average-based (original) center frequency update.

\begin{tabular}{c|c|c|c|c}
\hline SNR (dB) & $\mathbf{2 0}$ & $\mathbf{1 0}$ & $\mathbf{5}$ & $\mathbf{0}$ \\
\hline \hline GCS & $16 \pm 6$ & $54 \pm 48$ & $63 \pm 61$ & $79 \pm 79$ \\
\hline Average & $21 \pm 2$ & $73 \pm 45$ & $80 \pm 60$ & $114 \pm 106$ \\
\hline
\end{tabular}

\section{CONCLUDING REMARKS}

In this work, we presented the NA-MVMD method which augments the recently proposed MVMD method incorporating the noise injection paradigm, i.e. adding new channels with WGN. The NA-MVMD fully exploits the filter bank property of MVMD on WGN. The new method allows the processing of both univariate and multivariate signals making it a generalization of both VMD and MVMD. To evaluate its performance, NA-MVMD was applied to synthetic multicomponent signals showing better analytical ability, noise intolerance and less sensitivity in selecting the $K$ parameter. Moreover, an alternative center frequency update, i.e. a core calculation in MVMD algorithm, based on the GCS was proposed allowing faster convergence.

Nonetheless, the NA-MVMD should be subjected to more investigation regarding 1) the better understanding of the noise injection parameters, i.e. number of noisy channels and the noise amplitude, 2) the application to real-life univariate and/or multivariate signals and 3 ) the combination of the proposed paradigm with the other modifications/extensions of the original VMD and MVMD methods. 


\section{REFERENCES}

[1] Edward Bedrosian, "A product theorem for hilbert transforms," Proc. IEEE, vol. 51, pp. 868-869, 1963.

[2] Norden E Huang and et al., "The empirical mode decomposition and the hilbert spectrum for nonlinear and non-stationary time series analysis," Proceedings of the Royal Society of London. Series A: mathematical, physical and engineering sciences, vol. 454, no. 1971, pp. 903-995, 1998.

[3] Zhaohua Wu and Norden E Huang, "Ensemble empirical mode decomposition: a noise-assisted data analysis method," Advances in adaptive data analysis, vol. 1, no. 01, pp. 1-41, 2009.

[4] Jia-Rong Yeh, Jiann-Shing Shieh, and Norden E Huang, "Complementary ensemble empirical mode decomposition: A novel noise enhanced data analysis method," Advances in adaptive data analysis, vol. 2, no. 02, pp. 135-156, 2010.

[5] María E Torres and et al., "A complete ensemble empirical mode decomposition with adaptive noise," in 2011 IEEE international conference on acoustics, speech and signal processing. IEEE, 2011, pp. 4144-4147.

[6] Naveed ur Rehman and et al., "Emd via memd: multivariate noise-aided computation of standard emd," $A d$ vances in Adaptive Data Analysis, vol. 5, no. 02, pp. 1350007, 2013.

[7] Luan Lin, Yang Wang, and Haomin Zhou, "Iterative filtering as an alternative algorithm for empirical mode decomposition," Advances in Adaptive Data Analysis, vol. 1, no. 04, pp. 543-560, 2009.

[8] Jerome Gilles, "Empirical wavelet transform," IEEE transactions on signal processing, vol. 61 , no. 16, pp. 3999-4010, 2013.

[9] Ingrid Daubechies, Jianfeng $\mathrm{Lu}$, and Hau-Tieng $\mathrm{Wu}$, "Synchrosqueezed wavelet transforms: An empirical mode decomposition-like tool," Applied and computational harmonic analysis, vol. 30, no. 2, pp. 243-261, 2011.

[10] Georgios Apostolidis and Leontios Hadjileontiadis, "Swarm decomposition: A novel signal analysis using swarm intelligence," Signal Processing, vol. 132, pp. 40-50, 2017.

[11] Naveed Rehman and Danilo P Mandic, "Multivariate empirical mode decomposition," Proceedings of the Royal Society A: Mathematical, Physical and Engineering Sciences, vol. 466, no. 2117, pp. 1291-1302, 2010.
[12] Danilo P Mandic, Naveed ur Rehman, Zhaohua Wu, and Norden E Huang, "Empirical mode decompositionbased time-frequency analysis of multivariate signals: The power of adaptive data analysis," IEEE signal processing magazine, vol. 30, no. 6, pp. 74-86, 2013.

[13] Naveed ur Rehman and Hania Aftab, "Multivariate variational mode decomposition," IEEE Transactions on Signal Processing, vol. 67, no. 23, pp. 6039-6052, 2019.

[14] Antonio Cicone, "Multivariate fast iterative filtering for the decomposition of nonstationary signals," arXiv preprint arXiv:1902.04860, 2019.

[15] Omkar Singh and Ramesh Kumar Sunkaria, "An empirical wavelet transform based approach for multivariate data processing application to cardiovascular physiological signals," Bio-Algorithms and Med-Systems, vol. 14, no. $4,2018$.

[16] Alireza Ahrabian and et al., "Synchrosqueezing-based time-frequency analysis of multivariate data," Signal Processing, vol. 106, pp. 331-341, 2015.

[17] Xian-Bo Wang, Zhi-Xin Yang, and Xiao-An Yan, "Novel particle swarm optimization-based variational mode decomposition method for the fault diagnosis of complex rotating machinery," IEEE/ASME Transactions on Mechatronics, vol. 23, no. 1, pp. 68-79, 2017.

[18] Qiming Chen, Xun Lang, Lei Xie, and Hongye Su, "Detecting nonlinear oscillations in process control loop based on an improved vmd," IEEE Access, vol. 7, pp. 91446-91462, 2019.

[19] Jijian Lian, Zhuo Liu, Haijun Wang, and Xiaofeng Dong, "Adaptive variational mode decomposition method for signal processing based on mode characteristic," Mechanical Systems and Signal Processing, vol. 107, pp. 53-77, 2018.

[20] Yujie Zhao and et al., "A modified variational mode decomposition method based on envelope nesting and multi-criteria evaluation," Journal of Sound and Vibration, vol. 468, pp. 115099, 2020.

[21] Konstantin Dragomiretskiy and Dominique Zosso, "Variational mode decomposition," IEEE transactions on signal processing, vol. 62, no. 3, pp. 531-544, 2013.

[22] David Ramirez, Javier Via, and Ignacio Santamaria, "A generalization of the magnitude squared coherence spectrum for more than two signals: definition, properties and estimation," in 2008 IEEE International Conference on Acoustics, Speech and Signal Processing. IEEE, 2008, pp. 3769-3772.

[23] Dimitri P Bertsekas, Constrained optimization and Lagrange multiplier methods, Academic press, 2014. 